\title{
Performance Analysis of Metro WDM Star Network based on An Arrayed Waveguide Grating
}

\author{
Syed Abdul Mannan Kirmani, and Seoyong Shin* \\ Department of Communication Engineering, Myongji University, Yongin, 449-728, Korea \\ Hyo-Sik Yang \\ Department of Computer Engineering, Sejong University, Seoul, 143-747, Korea \\ Naeem Zafar Azeemi \\ Center for Advanced Studies in Telecommunication, COMSATS Institute of Information \\ Technology, Islamabad, Pakistan
}

(Received November 10, 2008 : revised December 14, 2008 : accepted December 15, 2008)

\begin{abstract}
In this paper, we analyze the performance of a reliable, scalable, and cost-effective switchless wavelength division multiplexing (WDM) network based on an arrayed waveguide grating (AWG). The network efficiency is significantly increased by spatially reusing wavelengths and exploiting multiple free spectral ranges (FSRs) of the AWG employed in the architecture. We have analyzed the performance of this architecture by simulating in Optsim ${ }^{\mathrm{TM}}$ with a control channel which exploits the broadband light source, i.e., an LED. The data was successfully transmitted to a distance of $30 \mathrm{~km}$ without using any EDFA's in the network, with a reasonable BER of $4.0530 \times 10^{-14}$. We have analyzed the performance of this architecture with a control channel which exploits the broadband light source, i.e., an LED, by simulating in Optsim ${ }^{\mathrm{TM}}$.
\end{abstract}

Keywords: Metro WDM Network, Arrayed Waveguide Grating, Spatial Reuse of Wavelength

OCIS codes : (060.0060) Fiber optics and optical communications; (060.4250) Networks; (060.4510) Optical communications

\section{INTRODUCTION}

Many new applications and services have emerged amidst the rapid growth of the internet and telecommunications industry resulting in a surge of data on voice networks [1]. This surge of data rendered the voice telecommunications infrastructure insufficient in the metropolitan area resulting in a metro gap. This dilemma provided us with a dire need to replace or upgrade the existing telecommunications infrastructure. So to cope with the changed realities and enable new applications and services to utilize huge bandwidths available in the long haul backbone networks, a metro WDM star network based on an arrayed waveguide grating is proposed and evaluated in $[2,3]$. The majority

*Corresponding author: sshin@mju.ac.kr of the earlier presented WDM network architectures are either based on a physical ring topology or a physical star topology [4] - [6]. It is shown that the AWG star networks clearly outperform the ring networks in terms of throughput, delay, and packet loss for unicast traffic with the expense of the single point of failure which could be overcome by redundancy [7]. The hardware parameter, i.e., degree of AWG and the number of FSRs (Free Spectral Ranges), and the software parameters, i.e., the number of slots per frame, the number of control slots in a frame, and retransmission probability, are optimized for a given network using a genetic algorithm [8]. AWG based star network can supplement the existing metro networks and increase their capacity to overcome the metro gap as shown in Fig. 1 [9].

In this paper, we are analyzing the performance of a star metro WDM network based on an Arrayed 


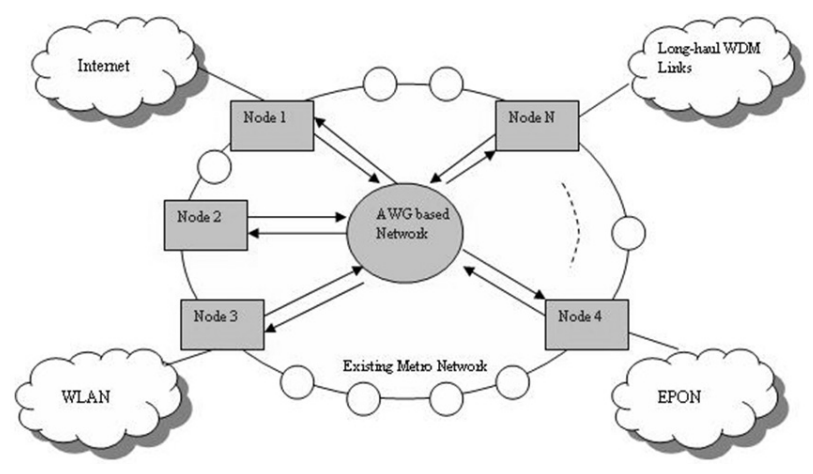

FIG. 1. Network architecture.

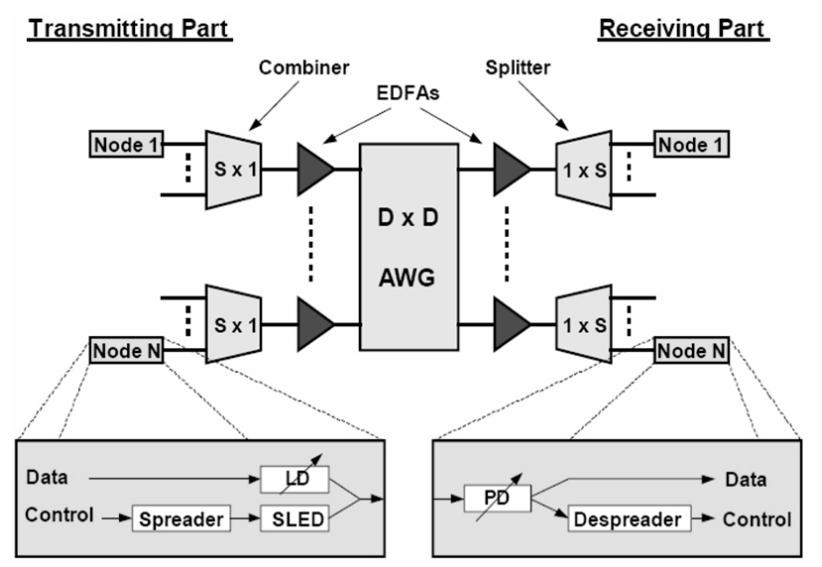

FIG. 2. Detailed network and node architecture.

Node (transmitting part)

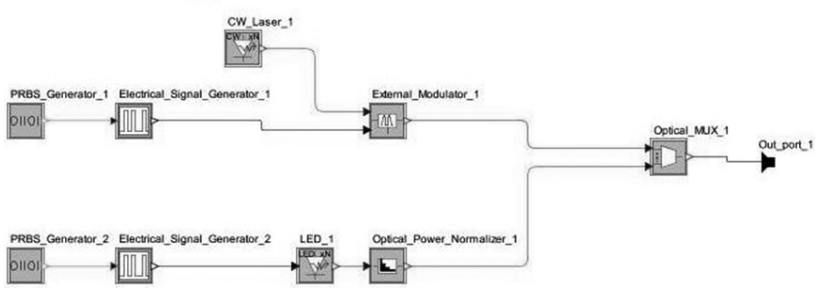

FIG. 3. Transmitting node architecture with in-band control channel simulated in OptsimTM

Waveguide Grating with control channel using a broadband light source, i.e., an LED (Light Emitting Diode) which was not considered in [3]. We measured a bit error rate (BER) as function of input power and measure the BER as function of distance between the source and the destination. As described in the numerical results section, the control channel does not significantly affect the performance of an AWG based network. The rest of the paper is organized as follows. In the following section we briefly describe the network and node architecture and the MAC protocol for the AWG based star network, and in Section III, the OptsimTM simulation results depicting the performance of the above mentioned network architecture is summarized. Section IV concludes the paper.

\section{NETWORK ARCHITECTURE}

Network architecture with MAC protocol for a AWG based star network is described in great detail in $[2$, 3]. In this section, we briefly describe it and give a concise insight into the architecture.

\subsection{Network and Node Architecture}

The basic architecture of the single-hop WDM star network is based on a $\mathrm{D} \times \mathrm{D}$ AWG, as shown in Fig. 2. Each node is connected to the network via two fibers, i.e., one for transmission and the other for reception. At each AWG input port, a wavelength-insensitive $\mathrm{S} \times 1$ combiner collects data from $\mathrm{S}$ attached nodes. Similarly, at each AWG output port, signals are distributed to $\mathrm{S}$ nodes by a wavelength-insensitive $1 \times \mathrm{S}$ splitter. Each node is equipped with a laser diode (LD) and a photodiode (PD) for data transmission and reception, respectively. Both data transmitter and receiver are tunable over $\lambda$ wavelengths which are not pre-assigned to nodes. The network connects $\mathrm{N}=\mathrm{D} \times \mathrm{S}$ nodes. At each $\mathrm{AWG}$ input port we exploit $\mathrm{R}$ adjacent free spectral ranges (FSRs) of the AWG. Each FSR consists of D contiguous wavelengths. The total number of wavelengths at each AWG input port is $\lambda=\mathrm{D} \times \mathrm{R}$. Note that the AWG allows for spatial wavelength reuse. As a result, the $\lambda$ wavelengths can be simultaneously applied at each of D AWG input ports, for a total of $\mathrm{D} \times \lambda$ wavelength channels connecting the D AWG input ports with the D AWG output ports. The MAC protocol makes use of a control channel to broadcast control information. The control channel is implemented as an in-band control channel by exploiting the spectral slicing of a broadband light source, i.e., Light Emitting Diode (LED), in conjunction with spectrum spreading of the control signals as shown in Fig. 3. Since in-band signaling is done so no additional receiver is required for the control channel. However, data and control information have to be distinguishable at the receiver by means of direct sequence spread spectrum techniques. The control information is spread before externally modulating the SLED. By using multiple spreading codes, several nodes are able to transmit multiple control packets at the same time, leading to code division multiple access (CDMA). CDMA not only allows simultaneous transmission of control packets but also provides some form of security [2].

\subsection{MAC Protocol}

The MAC protocol is needed to control the shared access to the medium by different nodes. It also handles the wavelength assignment to different nodes for communication. The control packets are broadcast in our network; hence they are received by all the nodes. In [11], it was shown that a normalized throughput (ratio of throughput and capacity) of up to $100 \%$ can be achieved if each node has global knowledge about all 
other nodes' activities. For this purpose each control packet has to be received by all the nodes as is the case in our network. In this way, receiver collisions of control packets are completely avoided and each node is able to check whether channel collision has occurred or not. Note that this approach works well as long as all nodes receive an intact copy of the control packet. All successful control packets are put in a distributed queue. Control packets are not required to have a field that indicates the wavelength of the corresponding data transmission. Instead, wavelengths can be assigned on a first-come-firstserved basis reducing the signaling overhead. In the MAC protocol, time is divided into cycles. Nodes are assumed to be synchronized to the cycle boundaries. Each cycle consists of D frames. Each frame contains $\mathrm{F}$ slots. The slot length is equal to the transmission time of a control packet. Each frame is partitioned into the first $\mathrm{M}, 1 \leq \mathrm{M}<\mathrm{F}$, slots and the remaining $\mathrm{F}-\mathrm{M}$ slots. For a detailed timing diagram, please refer $[2,3]$. In the first $\mathrm{M}$ slots, control signals are transmitted and all nodes must be tuned (locked) to one of the SLED slices carrying the control information. These $\mathrm{M}$ slots ado not have fixed assignments. Instead, control packets are sent on a contention basis using a modified version of slotted ALOHA.

\section{PERFORMANCE ANALYSIS}

We demonstrated the feasibility and the performance of our architecture by simulation in the commercially available optical communication systems software Optsim ${ }^{\mathrm{TM}}$. We simulated a 16 node architecture based on a $4 \times 4$ AWG, $4 \times 1$ combiner and $1 \times 4$ splitter. The $4 \times 4$ AWG used in simulation was designed in BEAMPROP ${ }^{\mathrm{TM}}$ simulation tool. The transmission graph of an input port (port 1) of designed AWG is shown in Fig. 4. The routing characteristics of the $4 \times 4$ AWG designed in BEAMPROP $^{\mathrm{TM}}$ are summarized in the Table 1.

We used just 4 wavelengths to communicate between 16 nodes simultaneously without any loss of data. This spatial wavelength reuse is possible because of the network architecture based on the AWG. Nodes attached to different AWG ports can use the same wavelength to communicate with other nodes without any channel collision. However, nodes attached to the same AWG port must use different wavelengths in order to avoid channel collision. We simulated the network with and without control channel to observe the decrease in BER due to the addition of a broadband control signal. The broadband signal for the control channel was generated from a $1550.4 \mathrm{~nm}$ LED with $0.1 \mathrm{~ns}$ of rise / fall time. The responsivity of the LED was $0.01 \mathrm{~A} / \mathrm{W}$ and the output power was $1 \mathrm{~mW}$. Since we are using CDMA for control channel, we do not need a high power LED. These parameter values correspond to an InGaAsP

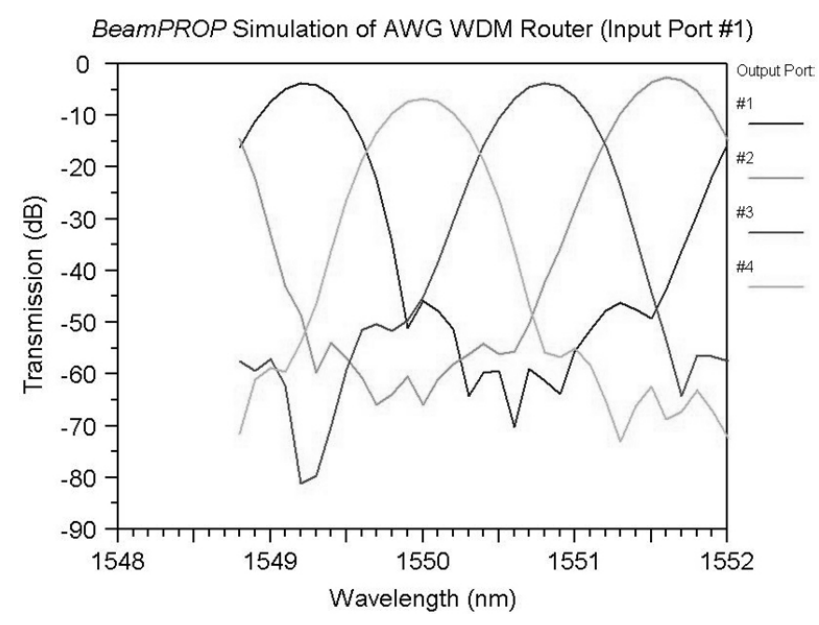

FIG. 4. Transmission Graph of input port (port 1) of AWG simulated in BEAMPROPTM

TABLE 1. Routing characteristics of $4 \times 4$ Arrayed Waveguide Grating (AWG) designed in BEAMPROP ${ }^{\mathrm{TM}}$ simulation

\begin{tabular}{c|c|c|c|c}
\hline \hline Input Port & 1 & 2 & 3 & 4 \\
\cline { 1 - 3 } Output Port & & & & \\
\cline { 1 - 4 } 1 & $\lambda_{1}$ & $\lambda_{4}$ & $\lambda_{3}$ & $\lambda_{2}$ \\
\hline 2 & $\lambda_{4}$ & $\lambda_{3}$ & $\lambda_{2}$ & $\lambda_{1}$ \\
\hline 3 & $\lambda_{3}$ & $\lambda_{2}$ & $\lambda_{1}$ & $\lambda_{4}$ \\
\hline 4 & $\lambda_{2}$ & $\lambda_{1}$ & $\lambda_{4}$ & $\lambda_{3}$ \\
\hline
\end{tabular}

TABLE 2. Important parameter values used for Optsim ${ }^{\mathrm{TM}}$ simulation

\begin{tabular}{l|l}
\hline \hline \multicolumn{1}{c|}{ Parameter } & \multicolumn{1}{c}{ Value } \\
\hline Number of Nodes & 16 \\
\hline Degree of AWG & 4 \\
\hline Degree of Combiner / Splitter & 4 \\
\hline Transmission Distance & $26 \mathrm{~km}$ \\
\hline Bit Rate (Data Channel) & $10 \mathrm{Gbps}$ \\
\hline Bit Rate (Control Channel) & $156.25 \mathrm{Mbps}$ \\
\hline LD Power & $10 \mathrm{~mW}$ \\
\hline LED Power & $1 \mathrm{~mW}$ \\
\hline LED wavelength & $1550.4 \mathrm{~nm}$ \\
\hline Node $1,5,9$ and 13 wavelength $\left(\lambda_{1}\right)$ & $1549.2 \mathrm{~nm}$ \\
\hline Node 2,6,10 and 14 wavelength $\left(\lambda_{2}\right)$ & $1550.0 \mathrm{~nm}$ \\
\hline Node 3,7,11 and 15 wavelength $\left(\lambda_{3}\right)$ & $1550.8 \mathrm{~nm}$ \\
\hline Node 4,8,12 and 16 wavelength $\left(\lambda_{4}\right)$ & $1551.6 \mathrm{~nm}$ \\
\hline
\end{tabular}

ELED [11]. Moreover, the bit rate for LED was needed to be a high because of CDMA. So, the bit rate chosen for the LED is $156.25 \mathrm{Mbps}$. The $3 \mathrm{~dB}$ modulation bandwidth of simulated LED is 275 Mbps. So, it is safe to modulate the LED at the chosen bit rate. Fig. 5 shows a plot of BER versus input power of the laser diode without in-band control channel. On the other 


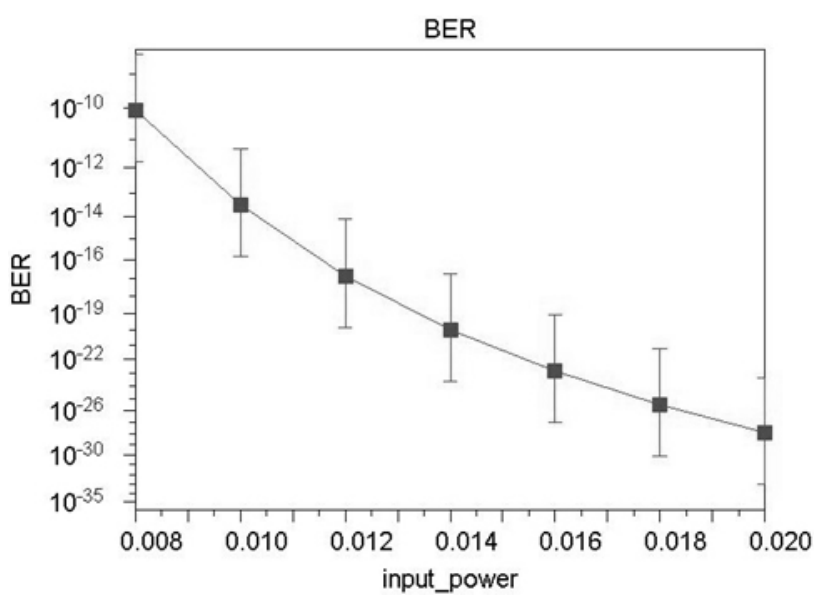

FIG. 5. Plot of BER versus Input Power (without inband control channel)

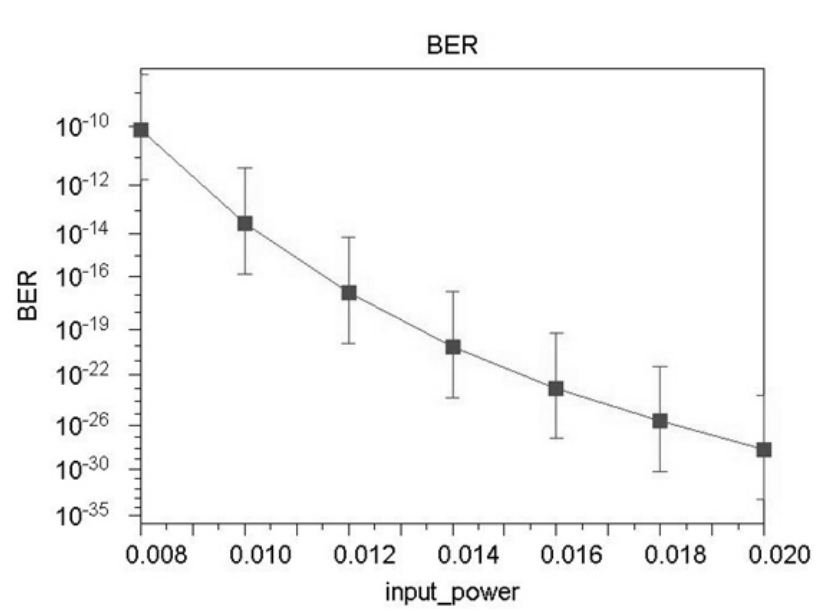

FIG. 6. Plot of BER versus Input Power (with in-band control channel)

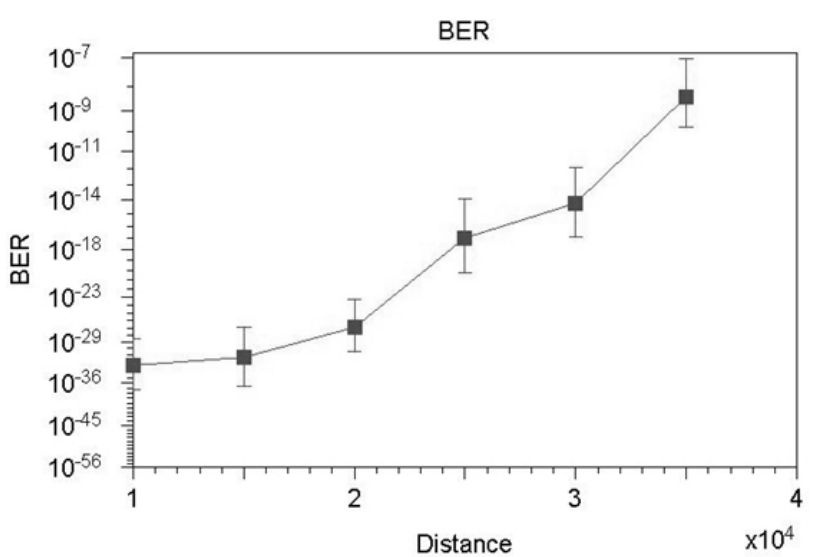

FIG. 7. Plot of BER versus Transmission Distance (without in-band control channel)

hand, Fig. 6 shows a plot of BER versus input power of the laser diode with in-band control channel. Similarly, Fig. 7 shows a plot of BER versus the transmission distance without any in-band control channel and Fig.

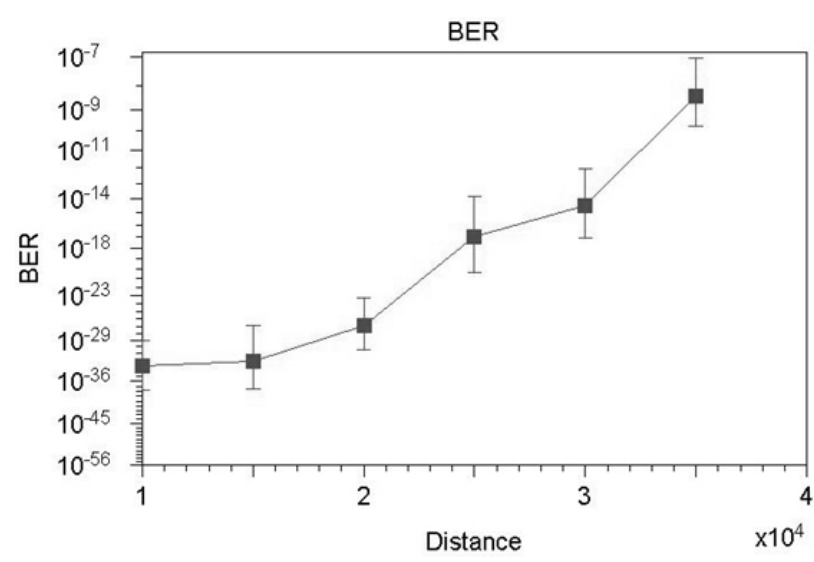

FIG. 8. Plot of BER versus Transmission Distance (with in-band control channel)

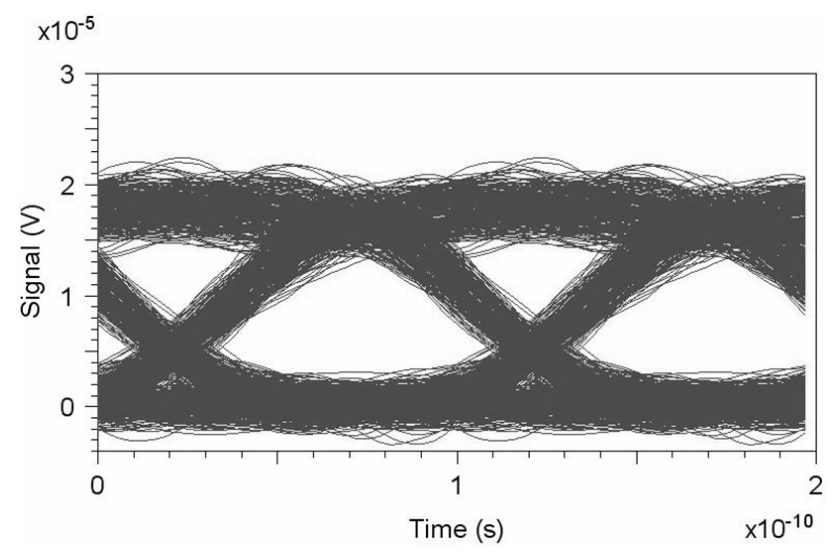

FIG. 9. Eye diagram of received signal at node 1

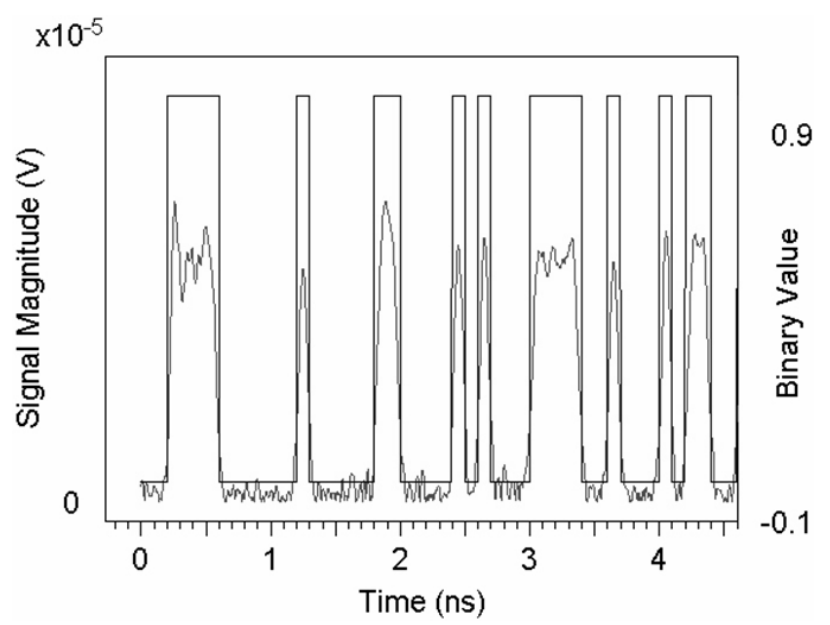

FIG. 10. Plot of binary data signal transmitted and the received electrical signal at node 1

8 shows a plot of BER versus the transmission distance with in-band control channel. It can be observed that the BER of the received data signal is not reduced much by adding the broadband control signal. The eye diagram of the received signal at node 1 is shown in the Fig. 
9. The Fig. 10 shows the plot of binary data signal transmitted and the electrical signal received at the receiver of node 1 . By adding in-band control channel the BER was increased from $3.2121 \times 10^{-14}$ to $4.0530 \times 10^{-14}$.

\section{CONCLUSION}

We have demonstrated the feasibility and the performance of the AWG based star network architecture. We observed that the signal can be transmitted successfully to a distance of $30 \mathrm{~km}$ with a reasonable BER of $4.0530 \times 10^{-14}$. This distance can be further increased by using optical amplifiers in the network. In our network 16 nodes can communicate using just 4 wavelengths at the same time. Moreover, this network is also scalable and cost-effective. Hence, this network is a good choice for either upgrading or installing a new metropolitan area network.

\section{ACKNOWLEDGMENT}

Authors would like to thank S. C. Kim for his kind help and advice throughout our research.

This work was supported by Korea Science and Engineering Foundation (KOSEF) grant funded by the Korean government (MOST) (No. R01-2006-000-10277-0).

Syed Abdul Mannan Kirmani was with Myongji University and is now with Center for Advanced Studies in Telecommunication, COMSATS Institute of Information Technology, Islamabad, Pakistan.

\section{REFERENCES}

[1] B. Mukherjee, "WDM Optical Communication Networks: Progress and Challenges," IEEE J. of Sel. Areas on Comm., vol. 18, no. 10, pp. 1810-1824, October 2000.

[2] S. A. M. Kirmani, S. Shin, H. S. Yang, "A Metro WDM Star Network with a Hybrid MAC Protocol Based on an Arrayed Waveguide Grating," J. Opt. Soc. Korea, vol. 11, no. 2, pp. 59-62, June 2007.

[3] M. Maier, M. Reisslein, and A. Wolisz, "A hybrid MAC protocol for a metro WDM network using multiple free spectral ranges of an arrayed waveguide grating," Computer Networks, vol. 41, no. 4, pp. 407-433, Mar. 2003.

[4] B. Mukherjee, "WDM - Based Local Lightwave Networks Part - I: Single - Hop Systems," IEEE Network Mag., vol. 6, no. 3, pp. $12-27$, May 1992.

[5] B. Mukherjee, "WDM - Based Local Lightwave Networks Part - II: Multihop Systems," IEEE Network Mag., vol. 6, no. 4, pp. 20 - 32, July 1992.

[6] M. Herzog, M. Maier, M. Reisslein, "Metropolitan Area Packet-Switched WDM Networks: A Survey on Ring Systems," IEEE Communications Surveys \& Tutorials, vol. 6, no. 2, pp. 2-20, Second Quarter, 2004.

[7] H. Yang, M. Herzog, M. Maier, M. Reisslein, "Metro WDM Networks: Performance Comparison of Slotted Ring and AWG Star Networks," IEEE J. of Sel. Areas on Comm., vol. 22, no. 8, pp. 1460-1473, October 2004.

[8] H. Yang, M. Maier, M. Reisslein, W. M. Carlyle, "A Genetic Algorithm based Methodology for Optimizing Multi-Service Convergence in a Metro WDM Network," IEEE J. Lightwave Tech., vol. 21, no. 5, pp. 1114-1133, May 2003.

[9] M. Maier, M. Herzog, M. Reisslein, “Topics In Optical Communications - STARGATE: The Next Evolutionary Step toward Unleashing the Potential of WDM EPONs," IEEE Comm. Mag., vol. 45, no. 5, pp. 50-56, May 2007.

[10] M. Chen, T.-S. Yum, "A conflict-free protocol for optical WDMA networks," in Proc. of IEEE Globecom, Phoenix, AZ, pp. 1276-1281, 1991.

[11] D. K. Mynbaev and L. L. Scheiner, Fiber Optic Communications Technology (Prentice Hall, 2001). 